\title{
Efficacy of electromagnetic lithotriptor in the treatment of renal and upper ureteral stones
}

\author{
Sadrollah Mehrabi ${ }^{*}{ }^{\circledR}$, Esmat Rasoli $^{2}$, Hamidreza Ghafarian Shirazi ${ }^{3}$, Amir Mehrabi ${ }^{2}$ \\ ${ }^{1}$ Clinical Research Development Unit, Yasuj University of Medical Sciences, Yasuj, Iran \\ ${ }^{2}$ Student Research Committee, Yasuj University of Medical Sciences, Yasuj, Iran \\ ${ }^{3}$ Faculty of Medicine, Yasuj University of Medical Sciences, Yasuj, Iran
}

\section{A R T I C L E I N F O}

Article Type:

Original

Article History:

Received: 5 February 2018

Accepted: 24 July 2018

Published online: 16 October 2018

Keywords:

Lithotriptor

Electromagnetic

Efficacy

Extracorporeal shock wave lithotripsy

Renal stone

Urinary tract stone

\begin{abstract}
A B S T RA C T
Introduction: The innovation of outpatient extracorporeal shock wave lithotripsy (ESWL) has created a revolution in the treatment of urinary tract stones.

Objectives: The aim of this study was to evaluate the success rate of electromagnetic lithotriptor, in the treatment of renal and upper ureteral stones.

Patients and Methods: In this study, 84 patients aged over 10 years, with renal and upper ureteral stones less than $20 \mathrm{~mm}$, were considered for ESWL. Patients were randomly assigned into two groups. In all patients, using ultrasound and fluoroscopy, stone was localized and using an electromagnetic machine by standard method, ESWL was performed with electromagnetic waves. ESWL started with $12 \mathrm{kV}$ and was increased to $18 \mathrm{kV}$, up to a maximum of 3500 shock waves. Two weeks later a kidney, ureter, and bladder (KUB) X-ray and ultrasonography was conducted and the success rate of lithotripsy according to the crushing of stones and decrease in stone size was measured and recorded.

Results: In this study, $61.9 \%$ of patients were male. The mean age of patients was $46.62 \pm 13.12$ years. The mean size of stones in both groups was $13.4 \pm 2.5 \mathrm{~mm}$. Around $56.3 \%$ of patients had opaque stones and $43.8 \%$ had non-opaque stones. A total of $63.5 \%$ of patients received up to 3000 shock waves and $37.5 \%$ of patients received more than 3000 shock waves. Complete efficacy and clearance of renal and ureteral stones was observed in $54.8 \%$ and $59.1 \%$ of cases, respectively $(P>0.05)$.

Conclusion: This study showed a similarity in the success and efficacy of electromagnetic lithotriptor in the treatment of renal and upper ureteral stones. Additionally, the success rate of opaque and non-opaque stones have no significant differences, while stone size and proportion of shock waves will not increase the efficacy of the electromagnetic lithotriptor in crushing the stones.
\end{abstract}

Implication for health policy/practice/research/medical education:

The innovation of outpatient extracorporeal shock wave lithotripsy (ESWL) has created a revolution in the treatment of urinary tract stones. It has been shown in different studies, that the success rate of extracorporeal lithotriptor depends on the location, size and opacity of stones and have been reported to be between $43 \%$ to $85 \%$. This study was performed on 84 patients with renal and upper ureteral stones less than $20 \mathrm{~mm}$. We found a similarity in the success rate and efficacy of an electromagnetic lithotriptor in the treatment of renal and upper ureteral stones. Additionally, no significant differences regarding opacity, size and location of stones in crushing the stones was seen.

Please cite this paper as: Mehrabi S, Rasoli E, Ghafarian Shirazi H, Mehrabi A. Efficacy of electromagnetic lithotriptor in the treatment of renal and upper ureteral stones. J Renal Inj Prev. 2019;8(2):82-85. DOI: 10.15171/jrip.2019.16.

\section{Introduction}

Urinary stone disease is a common disease in all societies and is the third most common urinary tract disease, after urinary tract infections and prostate disease. In
$20 \%$ of cases if not treated properly, urinary stones will lead to some degree of kidney dysfunction $(1,2)$. The prevalence of urinary tract stones is between $5 \%$ to $10 \%$ and it is more prevalent in men (2). The recurrence rate is 
estimated to be around 50\%. In the past decade, there has been an increase in the prevalence of urinary tract stones (3). The innovation of the outpatient extracorporeal shock wave lithotripsy (ESWL) has created a revolution in the treatment of urinary tract stones. At present, this modality is the treatment of choice for renal and ureteral stones less than 20-25 mm. It utilizes high-energy waves that are capable of passing through body tissues to crush stones into smaller pieces (2-4). For clearance of stones, the success rate of ESWL in one study was $95 \%$ for stones less than $1 \mathrm{~cm}$ and $88 \%$ in $1-2 \mathrm{~cm}$ stones of lower pole (5). The electromagnetic lithotriptor is one kind of electrohydraulic lithotriptor that has been marketed in the last decades $(6,7)$. The machine uses an isocentric imaging system that provides precise positioning and flat-out action. In different studies, the success rate of the extracorporeal lithotriptor depends on the location, size and opacity of stones, while it has been reported to be between 43 to $85 \%$ (5-10). However, studies on electromagnetic lithotripters are limited and have different results (6-8).

\section{Objectives}

This study aimed to evaluate the efficacy of electromagnetic lithotriptor in the treatment of renal and upper ureteral stones.

\section{Patients and Methods \\ Study design}

A total of 84 patients older than 10 years were selected for this study by simple sampling method. The patients had renal and upper ureteral stones less than $20 \mathrm{~mm}$ and were referred to a urology clinic and were candidates for ESWL (September 2014 to October 2015).

For all the patients, history, complete physical examination and basic serum samples including, PT, PTT, CBC, Na, $\mathrm{K}$, renal function tests ( serum $\mathrm{BUN}$ and creatinine), urinalysis and urine culture tests were conducted. Patients with severe cardiovascular or pulmonary disease, coagulation disorders, uncontrolled hypertension, pregnant women and those with any contraindications for analgesic and narcotics drugs were excluded. To conduct lithotripsy, all patients had IV line and were maintained in supine position. After adjusting the patient's position and bed, the stone was localized by ultrasonography and fluoroscopy. Using an electromagnetic machine, the standard procedure ESWL was conducted using electromagnetic shock waves. ESWL started with 12 kilovolts $(\mathrm{kV})$ and increased to $18 \mathrm{kV}$ in 10 minutes up to a maximum of 3500 shock waves. Oxygen saturation was controlled by pulse oximetry using a device during operation. After outpatient lithotripsy, the patients were observed for 2 hours. Patients without complications, were discharged with prescription of oral antibiotics and analgesic drugs. Two weeks later, the patients revisited and kidney, ureter, and bladder (KUB) and ultrasonography were done. Thereafter, the success rate of lithotripsy according to crushing of the stones and decrease in stone size were measured and recorded.

\section{Ethical issues}

The research followed the tenets of the Declaration of Helsinki. Informed consent was obtained. The research was approved by the ethical committee of Yasuj University of Medical Sciences (\# YUMS.1394.72).

\section{Statistical analysis}

All data were collected and analyzed by SPSS 18 software using descriptive (frequency, mean and standard deviation) and analytical (independent sample $t$ test and chi-square test) statistics and $P<0.05$ was considered as the level of significance.

\section{Results}

In this study, $61.9 \%$ of patients were male and $38.1 \%$ were female. The mean age of patients was $46.62 \pm 13.12$ years. Regarding the location of stones; $47.6 \%$ of patients had proximal ureteral stones and $52.4 \%$ of patients had kidney stones. The highest rate of stone was found in the renal pelvis whereas the lowest was in the lower calyxes. The size of the stones in $23.4 \%$ of the patients was between 5 to $10 \mathrm{~mm}$. In $42.2 \%$ of patients, it was between 10 and $15 \mathrm{~mm}$ and in $34.4 \%$ of patients, it was between 15 and $20 \mathrm{~mm}$. However, the overall average size of stones was $13.4 \pm 2.5 \mathrm{~mm}$. Regarding, opacity, $56.3 \%$ of patients had opaque stone while $43.8 \%$ had non-opaque stones. The duration of the procedure in $75 \%$ of patients was less than 40 minutes, and in $25 \%$ of patients it was more than 40 minutes. Around, $63.5 \%$ of the patients received up to 3000 shock waves and $37.5 \%$ of the patients received more than 3000 shock waves.

In $57.2 \%$ of cases, the efficacy of treatment has been perfect (complete crushing with the residue below $4 \mathrm{~mm}$ ), was relative (residue below $4 \mathrm{~mm}$ ) in $29.7 \%$ of the cases and was unsuccessful (non-crushing stones) in 13.1\%

Table 1. Overall efficacy of electromagnetic lithotriptor regarding rate of success

\begin{tabular}{lcc}
\hline Overall efficacy & No. of patients & Percent \\
\hline Complete & 48 & 57.2 \\
Relative & 25 & 29.7 \\
Failure & 11 & 13.1 \\
Sum & 84 & 100 \\
\hline
\end{tabular}

Table 2. Comparison of efficacy of electromagnetic lithotriptor in renal and ureteral stones

\begin{tabular}{lll}
\hline Overall efficacy & Renal & Upper ureter \\
\hline Complete (\%) & 64.3 & 59.1 \\
Relative (\%) & 21.4 & 18.2 \\
Failure (\%) & 14.3 & 22.7 \\
Sum (\%) & 100 & 100 \\
\hline
\end{tabular}


of the cases (Table 1). Complete efficacy and clearance of renal and ureteral stones was observed in $54.8 \%$ and $59.1 \%$ of cases, respectively $(P>0.05)$ (Table 2$)$. Therefore, the efficacy of treatment in terms of location and size of stones in the kidney and ureter was not significantly different. None of the patients required anesthesia and did not report any complications requiring hospitalization and intervention.

\section{Discussion}

Nowadays ESWL is the treatment of choice for most renal and upper ureteral calculi less than $20 \mathrm{~mm}$ with success rates of $60 \%-99 \%(11,12)$. The success rate of ESWL depends on several factors such as stone size, location, stone composition, as well as the type of lithotriptor (1317).

In this study, $65.6 \%$ of patients had renal stones while $34.4 \%$ of patients had proximal ureteral stones. Most of the stones were located in the renal pelvis while the lowest rates were in the lower calyx. In both groups, the mean size of stones was $13.4 \pm 2.5 \mathrm{~mm}$. Around $56.3 \%$ of patients had opaque stone and $43.8 \%$ of patients had nonopaque stones. A total of $63.5 \%$ of patients received up to 3000 shock and the remaining $37.5 \%$ of patients received more than 3000 shock waves. It was found that complete efficacy in renal and upper ureteral stones were $54.8 \%$ and $59.1 \%$, respectively. There was no significant difference in success rate regarding location, size and opacity of stones. To evaluate the efficacy of ESWL in the treatment of urinary stones, 1645 patients were treated with Dornier Compact Delta Electromagnetic Lithotriptor in the study conducted by Varshanidze et al. A total of $56.7 \%$ of patients were male and $43.3 \%$ were female. Of 1719 stones, $84.5 \%$ were opaque. They concluded that ESWL is the gold standard treatment of urinary stones less than $20 \mathrm{~mm}$ (18). Additionally, in a study by Elkholy et al, 97 patients (54 men, 43 women) with ureteral stones less than $10 \mathrm{~mm}$ were treated by ESWL. The patients had a mean age of 42.6 years and the mean shock numbers were 3125 shocks. All patients have some stone passage. They concluded that Dornier Compact 2 Electromagnetic Lithotriptor has a high efficacy in the treatment of ureteral stones without any major side effects (19).

Our results are consistent with these studies regarding sex distribution, mean age of patients and mean number of shock waves. Nonopaque stones are more in our study but there was no difference regarding success rate, opacity as well as shock numbers that are inconsistent with other studies. This may be due to technical skills, type of stones and the poor cooperation of patients during the procedure. For comparison of ESWL in the treatment of renal and ureteral stones, 183 patients were evaluated by Neisius et al. Around $46 \%$ of stones were in the kidneys and $54 \%$ were in the ureter while most of them were located in the renal pelvis (32\%). The mean stone size in the kidney and ureter was respectively 10 and $8 \mathrm{~mm}$. They concluded that stone free rate are higher in standard HM-3 lithotriptor. They found ESWL is the first line of treatment in urinary stones less than $10 \mathrm{~mm}$ while the rate of retreatment and complication is low (20). Our results are somewhat consistent with this study. However, the mean size of stones was larger in our study and $65.6 \%$ of stones were in kidneys. However, the success rate was lower in our study. In another study by White and Klein, 4621 patients with urinary stones were treated with the electromagnetic Delta Lithotriptor. The majority of stones were less than $10 \mathrm{~mm}$ and $74.4 \%$ of stones were in the kidney. The mean shock number was 2037 and the mean operation time was 23/8 minutes. The overall success rate during follow up was $85.1 \%(21)$.

The results of the study by White and Klein are inconsistent with our results while, the success rate in our study was $57.2 \%$ complete and $29.7 \%$ relative (residual stone more than $4 \mathrm{~mm}$ ). In addition, the mean time of ESWL was longer in our study. In fact, the lower efficacy and longer time of procedure in our study is somewhat due to larger and hard stones and more shock number and shorter time of follow up.

Likewise, Chung and Turney conducted ESWL in 130 patients with stones varying between $10-20 \mathrm{~mm}$ in size. Their overall success rate was $66.4 \%$. Accordingly, the success rate regarding stone location in the upper, lower and middle calyx were 65,64 and $70 \%$, respectively. They concluded that, the efficacy of ESWL for larger stones (10$20 \mathrm{~mm}$ ) are similar with smaller stones (22). Our results are similar with this study regarding overall efficacy, success rate and stone location but complete response in our study was lower than the study by Chung and Turney, which may be due to a shorter time of follow-up in our study.

\section{Conclusion}

The results showed a similarity in the success rate and efficacy of electromagnetic lithotriptor in the treatment of renal and upper ureteral stones. Additionally, no significant difference in the success rate of opaque and non-opaque stones crushing was detected. In addition stone size and number of shock waves do not affect efficacy.

\section{Limitations of the study}

Difficulty in localization of non-opaque ureteral stones and interference with bone density.

\section{Acknowledgments}

We present our appreciation to respected vice president technical and research department of Yasuj University of Medical Sciences that provided the facility to perform this project by their material and ethical support; also we thank all staff of ESWL ward of Shahid Beheshti hospital of Yasuj who assisted us in performing procedure. 


\section{Authors' contribution}

SM; the concept, design, data analysis, and manuscript preparation and final revision. ER; performing experiments and data collection and writing proposal. HGS; statistical analysis, manuscript editing, and manuscript review. AM, data collection and providing first draft and submission.

\section{Conflicts of interest}

None.

\section{Ethical considerations}

Ethical issues (including plagiarism, data fabrication, double publication) have been completely observed by the authors.

\section{Funding/Support}

This manuscript was extracted from M.D thesis of Esmat Rasoli (P.23.14.601) that supported financially by deputy of research of Yasuj University of Medical Sciences.

\section{References}

1. Stoller ML. Urinary stone disease. In: Tanago A, Mcaninch J, eds. Smith General Urology. 17th ed. New York: McGrawHill; 2008:246-277.

2. Chou YH, Li CC, Hsu H, Chang WC, Liu CC, Li WM, et al. Renal function in patients with urinary stones of varying compositions. Kaohsiung J Med Sci. 2011;27:264-7. doi: 10.1016/j.kjms.2010.11.008.

3. Basavaraj RD, Biyani CS, Anthony JB, Jon JC. The role of urinary kidney stone inhibitors and promoters in the pathogenesis of calcium containing renal stones. EAUEBU Update series. 2007;5:126-136. doi: 10.1016/j. eeus.2007.03.002

4. Mehrabi, S, Rahmani, A, Mehrabi, A, Motlagh, A. Extracorporeal shockwave lithotripsy versus laser lithotripsy by semirigid ureteroscope in treatment of upper ureteral stones. Acta Medica Medit. 2016;32:2075-8.

5. Nakamura K, Tobliume M, Narushima M, Yoshizava T, Nishikawa G, Kato Y, et al. Treatment of upper urinary tract stones with extracorporeal shock wave lithotripsy (ESWL) Sonolith vision. BMC Urol. 2011;11:26. doi: 10.1186/14712490-11-26.

6. Lalak NJ, Moussa SA, Smith G, Tolley DA. The Dornier Compact Delta Lithotripter: The First 500 Renal Calculi. J Endourol.2004;16:645-8.doi:10.1089/089277902753483637.

7. Pemberton RJ, Tolley DA. Comparisons of a newgeneration electroconductive spark lithotriptor and the Dornier Compact Delta for ureteral calculi in a quaternary referral center. J Endourol. 2006;20:732-6. doi: 10.1089/ end.2006.20.732.

8. Micali S, Sighinolfi MC, Grande M, Rivalta M, De Stefani S, Bianchi G. Dornier Lithotriptor S 220 F EMSE: the first report of over 1000 treatments. Urology. 2009;74:1211-4. doi: 10.1016/j.urology.2009.05.101.

9. Jones MN, Ranasinghe W, Cetti R, Newell B, Chu K,
Harper M, et al. Modified supine versus prone position in percutaneous nephrolithotomy for renal stones treatable with a single percutaneous access: a prospect randomized trial. Investig Clin Urol. 2016;57:268-73. doi: 10.4111/ icu.2016.57.4.268

10. Shoma AM, Eraky I, El-Kenawy MR, El-Kappany HA. Percutaneous nephrolithotomy in the supine position: technical aspects and functional outcome compared with the prone technique. Urology. 2002;60:388-92.

11. Lingeman JE, Newman D, Mertz JH, Mosbaugh PG, Steele RE, Kahnoski RJ, Coury TA, Woods JR. Extracorporeal shock wave lithotripsy: the Methodist Hospital of Indiana experience. J Urol. 1986;135:1134-7.

12. Rassweiler JJ, Renner C, Chaussy C, Thüroff S. Treatment of renal stones by extracorporeal shockwave lithotripsy: an update. Eur Urol. 2001;39:187-99.

13. Pareek G, Armenakas NA, Panagopoulos G, Bruno JJ, Fracchia JA. Extracorporeal shock wave lithotripsy success based on body mass index and Hounsfield units. Urology. 2005;65:33-6. doi: 10.1016/j.urology.2004.08.004

14. Choi JW, Song PH, Kim HT. Predictive factors of the outcome of extracorporeal shockwave lithotripsy for ureteral stones. Korean J Urol. 2012;53:424-30. doi: 10.4111/ kju.2012.53.6.424.

15. Argyropoulos AN, Tolley DA. Optimizing shock wave lithotripsy in the 21st century. Eur Urol. 2007;52:344-54. doi: $10.1016 /$ j.eururo.2007.04.066.

16. El-Nahas AR, El-Assmy AM, Mansour O, Sheir KZ. A prospective multivariate analysis of factors predicting stone disintegration by extracorporeal shock wave lithotripsy: the value of high-resolution noncontrast computed tomography. Eur Urol. 2007;51:1688-93. doi: 10.1016/j. eururo.2006.11.048

17. Mehrabi S, Manzori L, Gangi R, Mehrabi A. Comparison the success and complications of tubeless percutaneous nephrolithotomy in prone and supine position under spinal anesthesia. Acta Medica Mediterranea. 2016;32:2079-83.

18. Varshanidze LO, Tevzadze KG, Dzhincharadze GR, Managadze GL. [The efficacy of extracorporal shock wave lithotrypsy in urinary stone disease]. Georgian Med News. 2007;143:64-7. [Russian]

19. Elkholy MM, Ismail H, Abdelkhalek MA, Badr MM, Elfeky MM. Efficacy of extracorporeal shockwave lithotripsy using Dornier SII in different levels of ureteral stones. Urol Ann. 2014;6:346-51. doi: 10.4103/0974-7796.141003

20. Neisius A, Wöllner J, Thomas C, Roos FC, Brenner W, Hampel C, et al. Treatment efficacy and outcomes using a third generation shockwave lithotriptor. BJU Int. 2013;112:972-81. doi: 10.1111/bju.12159.

21. White W, Klein F. Five-year clinical experience with the Dornier Delta lithotriptor. Urology. 2006;68:28-32. doi:10.1016/j.urology.2006.01.031

22. Chung VY, Turney BW. The success of shock wave lithotripsy (SWL) in treating moderate-sized (10-20 mm) renal stones. Urolithiasis. 2016;44:441-4. doi: 10.1007/ s00240-015-0857-2.

Copyright ( $) 2019$ The Author(s); Published by Nickan Research Institute. This is an open-access article distributed under the terms of the Creative Commons Attribution License (http://creativecommons.org/licenses/by/4.0), which permits unrestricted use, distribution, and reproduction in any medium, provided the original work is properly cited. 